\title{
SINGULAR PERTURBATION THEORY FOR SEMIBOUNDED OPERATORS
}

BY W. M. GREENLEE

Communicated by H. F. Wein berger, May 1, 1975

In this announcement an operator theoretic approach to singular perturbation expansions for simple eigenvalues is outlined. Corresponding results hold for eigenvectors, eigenvalues of finite multiplicity, and spectral concentration near eigenvalues of finite multiplicity.

We seek first and second order approximations for problems to which the regular perturbation method does not apply (cf. [2]). First presented are the abstract singular perturbation expansions, followed by mention of some results for differential problems. Application of the abstract results to concrete problems is involved, but our framework makes Lions' method of correctors [3], as well as boundary layer techniques (cf. [4]), applicable to eigenvalue problems.

Let $H$ be a complex Hilbert space with inner product $(v, w)$ and norm $|v|$. Let $b(v, w)$ be a Hermitian symmetric bilinear form defined on a linear manifold $D(b)$ which is dense in $H$. We assume that the quadratic form corresponding to $b(v, w)$ has a positive lower bound, and is closed. Then $D(b)$, with inner product $b(v, w)$, is a Hilbert space. Further let $a(v, w)$ be a Hermitian symmetric bilinear form defined on a linear manifold $D(a)$ which is dense in $D(b)$, and assume that the quadratic form corresponding to $a(v, w)$ is nonnegative, and closed in $D(b)$.

Let $B$ be the positive definite selfadjoint operator in $H$ defined by $(B v, w)$ $=b(v, w)$ for all $w \in D(b)$, on $D(B)=\{v \in D(b): w \rightarrow b(v, w)$ is continuous on $D(b)$ in the topology induced by $H\}$. Similarly let $A_{\epsilon}, \epsilon>0$, be the positive definite selfadjoint operator in $H$ defined by $\left(A_{\epsilon} v, w\right)=\epsilon a(v, w)+b(v, w)$, and A the nonnegative selfadjoint operator in $D(b)$ defined by $b(A v, w)=a(v, w)$.

Assume that $\lambda$ is an isolated simple eigenvalue of $B$ with corresponding eigenvector $u$ normalized in $H$. Assume further that $\lambda$ is stable under the above perturbation, i.e., that for $\epsilon$ sufficiently small the intersection of any isolating interval for $\lambda$ and the spectrum of $A_{\epsilon}$ consists of a single simple eigenvalue $\lambda_{\epsilon}$ of $A_{\epsilon}, \lambda_{\epsilon} \rightarrow \lambda$ as $\epsilon \downarrow 0$ (cf. [2]).

Now let $\eta=\left(A_{\epsilon}^{-1} u, u\right)$. It is easily shown that $\eta=\lambda^{-1}-\epsilon \lambda^{-2} \lambda_{\epsilon}^{\prime}$, where $\lambda_{\epsilon}^{\prime}=b\left(\mathrm{~A}(\epsilon \mathrm{A}+I)^{-1} u, u\right)$ and that $\epsilon \lambda_{\epsilon}^{\prime} \rightarrow 0$ as $\epsilon \downarrow 0$ (cf. [1]).

THEOREM 1. (i) $\lambda_{\epsilon}=\lambda+O\left(\epsilon \lambda_{\epsilon}^{\prime}\right)$ as $\epsilon \rightarrow 0$.

AMS (MOS) subject classifications (1970). Primary 47A55, 35B25. 
(ii) If there exists a Borel function $f:(0, \infty) \rightarrow[1, \infty)$ such that $f(\mu) \rightarrow$ $\infty$ as $\mu \rightarrow \infty$ and $D(B) \subset D(f(A))$, the inclusion being both algebraic and topological with the respective graph norms, then $\lambda_{\epsilon}=\lambda+\epsilon \lambda_{\epsilon}^{\prime}+o\left(\epsilon \lambda_{\epsilon}^{\prime}\right)$ as $\epsilon \downarrow 0$.

(iii) If the hypothesis of (ii) is satisfied by $f(\mu)=(\mu+1)^{\tau}$ where $0<$ $\tau<1 / 2$, then $\lambda_{\epsilon}=\lambda+\epsilon \lambda_{\epsilon}^{\prime}+O\left(\epsilon^{2 \tau+1} \lambda_{\epsilon}^{\prime}\right)$ as $\epsilon \downarrow 0$.

Recall that if $u \in D\left((A+I)^{1 / 2}\right)=D(a)$, the regular perturbation method applies to first order for $\lambda_{\epsilon}$. Also observe that part (iii) of Theorem 1 may give more than the first order correction to $\lambda_{\epsilon}$, depending on the number of terms in an asymptotic expansion of $\epsilon \lambda_{\epsilon}^{\prime}$ which are of lower order than $\epsilon^{\mathbf{2}+1} \lambda_{\epsilon}^{\prime}$. One way to investigate the asymptotic behaviour of $\epsilon \lambda_{\epsilon}^{\prime}$ is to find $k:\left(0, \epsilon_{0}\right] \rightarrow(0, \infty)$, $\kappa(\epsilon) \longrightarrow 0$ as $\epsilon \downarrow 0$, and $0 \neq u^{\prime} \in H$ such that

(iv) $\mid \epsilon \mathrm{A}\left(\epsilon \mathrm{A}+I^{-1} u-\kappa(\epsilon) u^{\prime} \mid=o(\kappa(\epsilon))\right.$ as $\epsilon \downarrow 0$, which implies that $\epsilon \lambda_{\epsilon}^{\prime}=$ $\kappa(\epsilon) \lambda\left(u^{\prime}, u\right)+o(\kappa(\epsilon))$ as $\epsilon \downarrow 0$.

THEOREM 2. If there exist $\kappa, u^{\prime}$ for which (iv) holds, then

$$
\lambda_{\epsilon}=\lambda+\epsilon \lambda_{\epsilon}^{\prime}-\kappa^{2}(\epsilon) \lambda\left(S u^{\prime}, u^{\prime}\right)+o\left(\kappa^{2}(\epsilon)\right) \text { as } \epsilon \downarrow 0,
$$

where $S$ is the bounded selfadjoint operator in $H$ defined by $S u=0, S=$ $B(B-\lambda)^{-1}$ on $\{u\}^{\perp}$.

This provides a generalization of the second order regular perturbation formula. When (iv) holds the first order correction to $u$ is $-\kappa(\epsilon) S u^{\prime}$; but observe that (v) yields a classical asymptotic expansion of $\lambda_{\epsilon}$ through order $\kappa^{2}(\epsilon)$ only if $\epsilon \lambda_{\epsilon}^{\prime}$ can be expanded through this order.

Theorems 1 and 2 apply to the usual higher derivative singular perturbations of differential eigenvalue problems (cf. [5], [4]). For such problems $k(\epsilon)=\epsilon^{2 \sigma}$ where $\sigma$ is the supremum of those $\tau$ for which the hypothesis of part (iii) of Theorem 1 holds (cf. [1]). Also, (iv) corresponds to finding the first correction in the outer expansion.

EXAMPLE. We list explicit conclusions for a different type of singular perturbation problem on the interval $[0, l]$.

$$
\begin{gathered}
-y_{\epsilon}^{\prime \prime}+\left[a x^{-2}+V(x)+\epsilon x^{-2 \alpha}\right] y_{\epsilon}=\lambda_{\epsilon} y_{\epsilon}, \\
y_{\epsilon}(0)=y_{\epsilon}(l)=0,
\end{gathered}
$$

where $^{\prime}=d / d x, \alpha>1, a>-1 / 4$ and, for simplicity, $V \in C_{0}^{\infty}(0, l)$. By adding a multiple of the identity operator to both sides, this problem fits into our framework with $H=L^{2}(0, l)$. The corresponding problem on $(0, \infty)$ would be a hard core potential perturbation of the radial equation of quantum mechanics. With $\beta=1 / 2(\alpha-1)$ and $\gamma=\beta(1+4 a)^{1 / 2}$, part (iii) of Theorem 1 yields the first order results: 


$$
\lambda_{\epsilon} \sim \begin{cases}\lambda+\lambda_{1} \epsilon^{\gamma} & \text { if } 0<\gamma<1 \text { and } \gamma<2 \beta \\ \lambda+\lambda_{1} \epsilon \log \epsilon & \text { if } \gamma=1 \text { and } 1 \leqslant 2 \beta \\ \lambda+\lambda_{1} \epsilon & \text { if } \gamma>1 \text { and } 1<2 \beta \\ \lambda+\lambda_{1} \epsilon^{2 \beta} & \text { otherwise, }\end{cases}
$$

where $\lambda_{1}$ is independent of $\epsilon$. Second order results follow from Theorem 2 if $0<\gamma \leqslant 1$ and $\gamma<2 \beta$, or if $\gamma>1$ and $2<\gamma+2 \beta$. In the other cases, i.e., $0<\gamma \leqslant 1$ and $\gamma \geqslant 2 \beta$, or $\gamma>1$ and $2 \geqslant \gamma+2 \beta$, while (iv) remains unverified, second order results are obtainable from part (iii) of Theorem 1.

\section{REFERENCES}

1. W. M. Greenlee, Singular perturbation of eigenvalues, Arch. Rational Mech. Anal. 34 (1969), 143-164. MR 40 \#3038.

2. T. Kato, Perturbation theory for linear operators, Die Grundlehren der math. Wissenschaften, Band 132, Springer-Verlag, New York, 1966. MR 34 \#3324.

3. J. L. Lions, Perturbations singulières dans les problèmes aux limites et en contrôle optimal, Lecture Notes in Math., vol. 323, Springer-Verlag, Berlin, 1973.

4. R. E. O'Malley, Jr., Topics in singular perturbations, Advances in Math. 2 (1968), 365-470. MR $38 \# 382$.

5. M. I. Višik and L. A. Ljusternik, Regular degeneration and boundary layer for linear differential equations with a small parameter, Uspehi Mat. Nauk 12 (1957), no. 5 (77), 3-122; English transl., Amer. Math. Soc. Transl. (2) 20 (1962), 239-364. MR 20 \#2539; $25 \# 322$.

DEPARTMENT OF MATHEMATICS, UNIVERSITY OF ARIZONA, TUCSON, ARIZONA 85721 